\title{
Study of apparent molal volume and viscosity of mutual citric acid and disodium hydrogen orthophosphate aqueous systems
}

\author{
MAN SINGH \\ Chemistry Research Lab, Deshbandhu College, University of Delhi, New Delhi 110019 \\ e-mail: mansingh50@hotmail.com
}

MS received 24 March 2004; revised 14 December 2005

\begin{abstract}
Fundamental properties, density $(\rho)$ and viscosity $(\eta)$, of citric acid (CA) and disodium hydrogen orthophosphate (DSP) at various strengths were obtained at different temperatures. The $\rho$ and $\eta$ values were used to determine apparent molal volumes and viscosity of systems. The $\rho, V_{\phi}$ and $\eta$ values were regressed against molality $m$ for $\rho^{0}, \eta^{0}$ and $V_{\phi}^{0}$, the limiting constants at infinite dilution $(m \rightarrow 0)$ for ionic and molecular interactions. The $\rho^{0}$ and $V_{\phi}^{0}$ of aq. acids are higher than those of aq. DSP and the viscosity of DSP is higher than that of aq. CA. Examination of $\rho^{0}$ and $V_{\phi}^{0}$ functions indicates that mutual compositions of $\mathrm{CA}$ and DSP counterbalance concentration and temperature effects on $\mathrm{pH}$ in bioprocesses.
\end{abstract}

Keywords. Citric acid-disodium hydrogen orthophosphate aqueous system; density/viscosity of aqueous systems; limiting constants at infinite dilution.

\section{Introduction}

Mutually citric acid (CA) and disodium hydrogen orthophosphate (DSP) have been useful buffers for studying biosystems, ${ }^{1,2}$ as physiological and biological activities are medium-dependent. Thus study of their interactions and physical characterization is essential. As per molecular modelling data, their interactions with water could influence actual biological processes and hence estimation of parameters such as density, volume and viscosity become necessary. CA and DSP are solvated in aqueous solution, occupying certain volume due to dipolar and ionic forces between them. This may have implications on the capacity and range of buffer usage. Also, CA is an important compound in cellular respiration for oxidizing glucose, fatty and amino acids and is related to acetyl coenzyme A of Kreb's cycle. ${ }^{3}$ Both CA and its conjugate base DSP of intra/extracellular fluids act as a first line of defence against changes of the internal $\mathrm{pH}^{4-7}$ As molar expansion/contraction affects the interaction of buffers with biofluids/ biochemicals, $\rho$ and $V_{\phi}$ data in particular help elucidate structural interactions and reorientations with the substrate ${ }^{8,9}$ and depict the state of hydration, transition pairwise interaction ${ }^{10-12}$ and electrostatic force $^{13-18}$ as a function of temperature and composition of bioactive molecules.

\section{Experimental}

Citric acid (AR, BDH) and disodium hydrogen orthophosphate (AR, E Merck) were dried, stored in a $\mathrm{P}_{2} \mathrm{O}_{5}$ vacuum dessicator and used by weighing out $(w / w)$ for making solutions in deionised, tripledistilled with $\mathrm{KMnO}_{4}$ and $\mathrm{KOH}$ and degassed (by boiling) water of $1 \times 10^{-7} \Omega$ conductance. Densities and flow times were measured with bicapillary pyknometer (of $20 \mathrm{ml}$ volume and $20 \cdot 22456 \mathrm{~g}$ ) and low shear Ubhelohde viscometer with $25 \mathrm{ml}$ solution bulb with $\pm 0.01 \mathrm{~K}$ control in temperature at $1 \mathrm{~atm}$ pressure. Efflux time was measured with a digital electronic racer with $\pm 1.0 \times 10^{-2} \mathrm{~s}^{-1}$ accuracy. Pyknometer weights were obtained with a $\pm 0.01 \mathrm{mg}$ Dhona balance model 100 DS and solutions were thermostated for $30 \mathrm{~min}$ prior to weighing. Temperature was maintained by circulating cold water from a cooling machine, MK70 GDR, through a thick-walled plastic tube insulated with asbestos thread. A parallel heating assembly with electronic relay encircuited with a thermometer after a 5-watt heating immersion rod were used. Temperature was checked with a calibrated Beckman thermometer (Thermal Division, NPL, New Delhi). The pyknometer was calibrated with aq. $\mathrm{NaCl}$ and the viscometer with water. 


\section{Results and discussion}

The $\rho$ was calculated as

$$
\begin{aligned}
\rho= & \mathrm{wt}(\text { solution } / \mathrm{wt}(\text { solvent }) \\
& +0 \cdot 0012(1-\mathrm{wt}(\text { solution } / \mathrm{wt}(\text { solvent })) .
\end{aligned}
$$

Densities of the solution $(\rho)$ and solvent $\left(\rho_{0}\right)$ the buoyancy correction for air $(0.0012 \quad(1-\mathrm{wt}$ (solution/wt(solvent), molality (m) and weights (wt) were measured with the pyknometer. The error in $\rho$ is calculated by statistical methods, ${ }^{19} V_{\phi}$ is computed $^{9}$ from the equation,

$$
V_{\phi}=1 / \rho\left(M-(1000 / m)\left(\left(\rho-\rho_{0}\right) /\left(\rho_{0}\right)\right) .\right.
$$

$M$ is the molar mass of solute, an uncertainty in $V_{\phi}$ is computed from the equation,

$$
V_{\phi}=(1000 / m) \Delta \rho .
$$

$\Delta \rho=\rho-\rho_{0}, \rho_{0}$ is solvent density, viscosity $\eta$ is calculated from the relation,

$$
\eta=(\rho \cdot t) /\left(\rho_{0} \cdot t_{0}\right) \cdot \eta_{0} .
$$

$t$ and $t_{0}$ are flow times, $\eta$ and $\eta_{0}$ are viscosities of solution and solvent respectively. The error in $\eta$ was obtained like $\rho$, the $\rho$ data are regressed as given below,

$$
\rho=\rho^{0}+S_{d} m .
$$

$\rho^{0}$ is the limiting density at $m \rightarrow 0, S_{d}$ is the slope and $V_{\phi}$ values are fitted as,

$$
V_{\phi}=V_{\phi}^{0}+S_{v} m \text {. }
$$

$V_{\phi}^{0}$ is the limiting value of $V_{\phi} . V_{\phi}$ is a measure of the ion-solvent and $S_{v}$ of the ion-ion interactions. The $\eta$ values were fitted as $\eta=\eta^{0}+A_{v} m$, where $\eta^{0}$ is the limiting viscosity and $A_{v}$ the slope. $\rho$ vs $m$ of DSP in aq. CA and vice versa are plotted in figures 1 and 2 , and $\eta$ of the aq. systems in figure 3. $\rho^{0}$ and $\eta$ vs $m$ are plotted in figure 4. $\eta^{0}$ vs $T, \eta^{0}$ vs $m$ of aq. CA and DSP, $\rho^{0}$ vs $m$ CA and DSP are shown in figure 5.

\section{Binary systems}

The $\rho$ values of 0.050 to $1.25 \mathrm{~mol} \mathrm{~kg}^{-1}$ aq. $\mathrm{NaCl}$ were measured for $V_{\phi}^{0}$ reproduced to $\pm 0 \cdot 05 / 10^{-6} \mathrm{~m}^{3} \mathrm{~mol}^{-1}$, which compares well with the literature ${ }^{18}$ values. Values of water density ${ }^{19 a}$ and viscosity ${ }^{19 b}$ were taken from literature: an increase and decrease in $\rho$ with concentration and temperature respectively, implies formation and breakage of hydrogen bonds. The $\rho^{0}$ values of aq. DSP and CA systems are found higher than that of water by $0.00016,-0 \cdot 00126,0.00188,-0 \cdot 0003$ and $0.00021,-0.00024,0.0015,0.00014 / 10 \mathrm{~kg} \mathrm{~m}^{-3}$ at $298 \cdot 15,303 \cdot 15,308 \cdot 15$ and $313 \cdot 15 \mathrm{~K}$, respectively (figures 1 and 2). A larger decrease in $\rho^{0}$ of DSP with temperature compared to CA is noted which implies that the ion-solvent interaction of DSP in water compared to CA with water is affected more by thermal energy. Thermal energy seems to weaken electrostatic forces in water. The values indicate slightly stronger molecular interaction of CA than that of DSP and this is, perhaps, due to stronger hydrophilic interaction of undissociated CA with water. The $\eta^{0}$ values of aq. DSP are seen in a range of 0.96422 to 0.59344 from $298 \cdot 15$ to $313.15 \mathrm{~K}$ and those of CA from 0.79643 to $0.64677 / 0 \cdot 1 \mathrm{~kg} \mathrm{~m}^{-1} \mathrm{~s}^{-1}$. The $\eta^{0}$ of aq. DSP is higher than that of aq. CA by

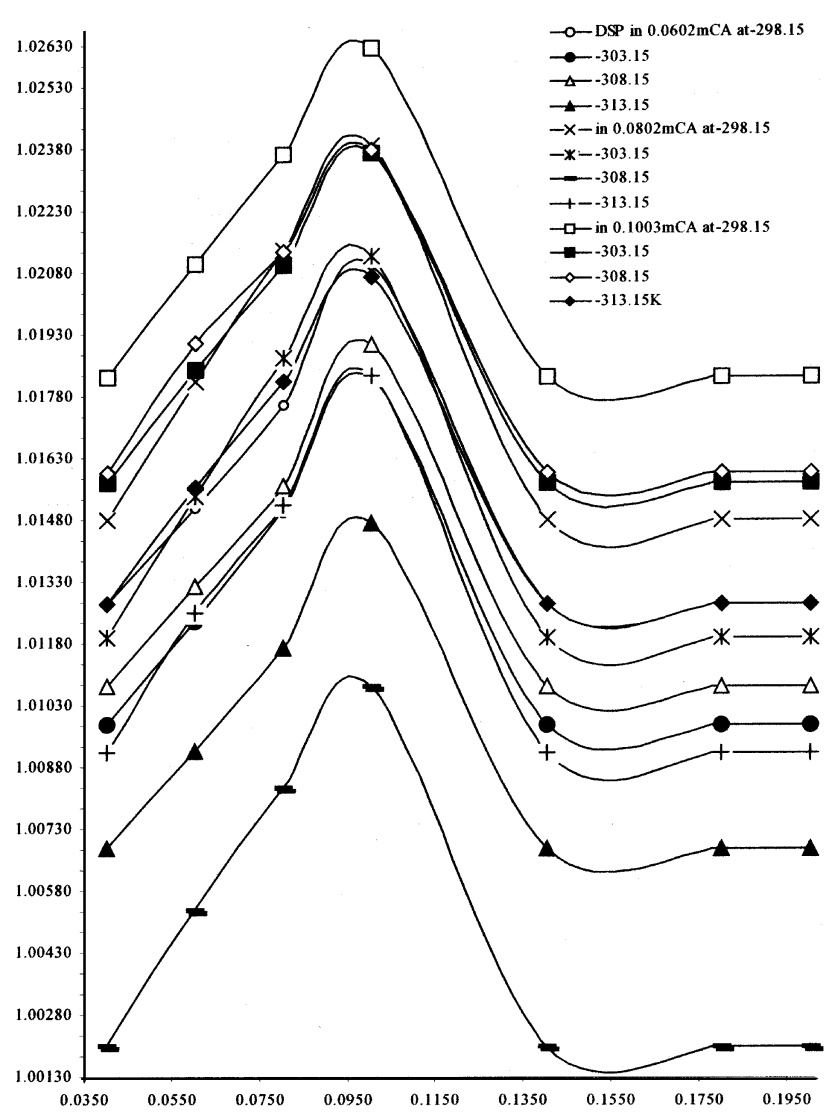

Figure 1. Density vs $m$ of DSP systems in variable compositions of $\mathrm{CA}$ at four different temperatures. 
$0 \cdot 16779$ (figure 3) and the value decrease with temperature. These values denote larger frictional forces on adjacent laminar flow for ion-hydration producing higher values of $H^{0}$. Therefore an ionic solution of DSP could be termed rigid a viscous medium while that of CA a soft medium. A larger decrease in $\eta^{0}$ of DSP than that of CA with temperature seriously destabilizes ion-solvent interactions in DSPwater. The slope constant $A_{v}$ of $\eta^{0}$ for DSP is found to be between 0.49611 and 0.82629 and that for aq. CA from $2 \cdot 20745$ to $0 \cdot 17170 / 0 \cdot 1 \mathrm{~kg} \mathrm{~m}^{-1} \mathrm{~s}^{-1}$. The $V_{\phi}^{0}$ values of DSP are obtained in the range 51.37 to 65.46 against those of CA from 101.64 to $106 \cdot 02 / 10^{-6}$ $\mathrm{m}^{3} \mathrm{~mol}^{-1}$. This indicates that ion-water interactions cause greater internal pressure that seems responsible for contraction in volume while in aq. DSP, CAwater leads to weakening of interaction lowering pressure and producing higher $V_{\phi}^{0}$ values. For both the systems, $V_{\phi}^{0}$ values increase with rise in temperature from $298 \cdot 15$ to $303 \cdot 15$ by about 36 and $13 \cdot 6 / 10^{-6}$ $\mathrm{m}^{3} \mathrm{~mol}^{-1}$ for DSP and CA and in the range $303 \cdot 15$ to $308.15 \mathrm{~K}, \quad V_{\phi}^{0}$ decreases by 54.89 and $17.6 / 10^{-6}$ $\mathrm{m}^{3} \mathrm{~mol}^{-1}$ for DSP and CA respectively. It certifies

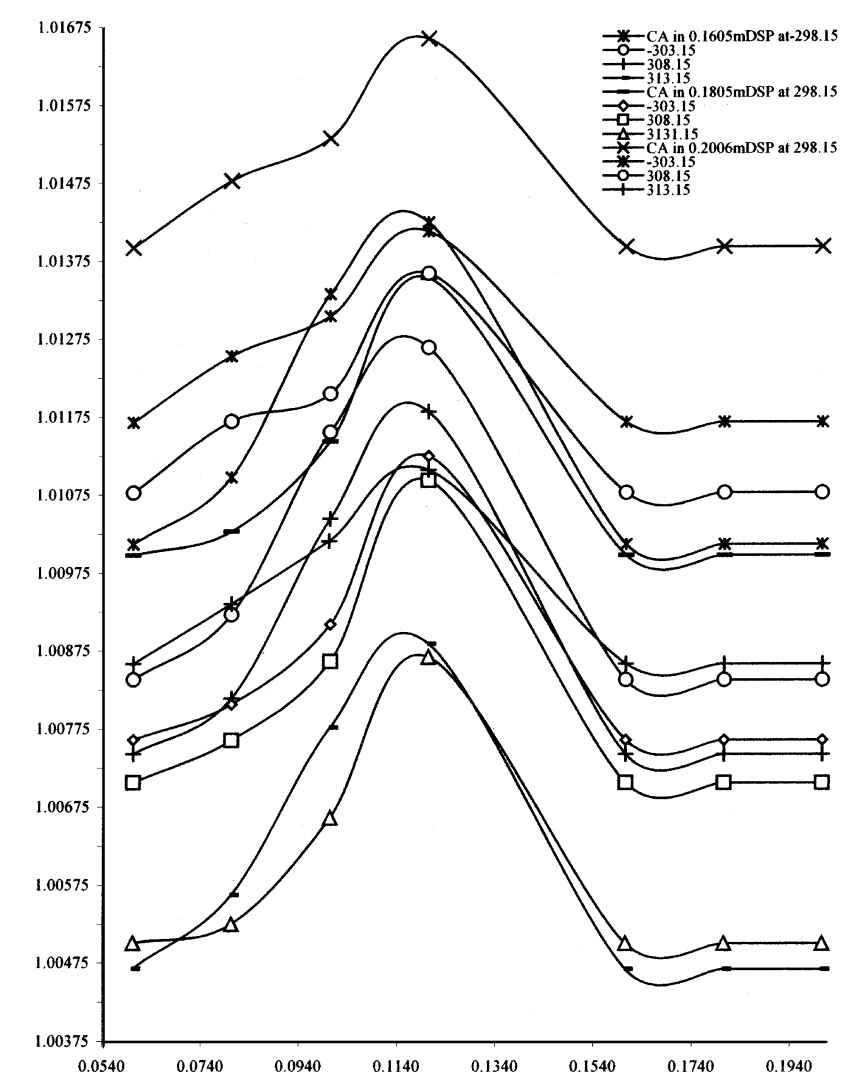

Figure 2. Density vs $m$ of CA in variable compositions of DSP at four temperatures. that CA introduces an element of thermodynamic stability, due to molecular interactions with water, unlike the ionic DSP. It is important to note that DSP has more effect on transport functions which may be due to ion-ion interaction rather than ion-solvent effects.

\section{Ternary system}

The $\rho$ values of DSP in variable composition of CA in water increase from 0.035 to 0.0910 smoothly, then rapidly to $0 \cdot 1335 \mathrm{~mol} \mathrm{~kg}^{-1}$ and finally decrease, after 0.1335 to $0.1895 \mathrm{~mol} \mathrm{~kg}^{-1}$ at a steep slope (figure 1). DSP systems have maximum values at around $0.0910 \mathrm{~mol} \mathrm{~kg}^{-1}$. Similar pattern of $\rho$ data for CA in variable composition of DAP in water is noted and depicted in figure 2 with maximum at $0.114 \mathrm{~mol} \mathrm{~kg}^{-1}$. Notably slopes before and after these compositions are very close and we conclude the same order of composition influence of CA on DSP and vice versa. The higher $\rho$ values are reported for DSP in $0.1003 \mathrm{~m} \mathrm{CA}$ at 298.15 and lower values for DSP in $0.0802 \mathrm{~m} \mathrm{CA}$ at $313.15 \mathrm{~K}$. The maximum $\rho$ value is noted for 0.095 of CA and $0.114 \mathrm{~mol} \mathrm{~kg}^{-1}$ of DSP

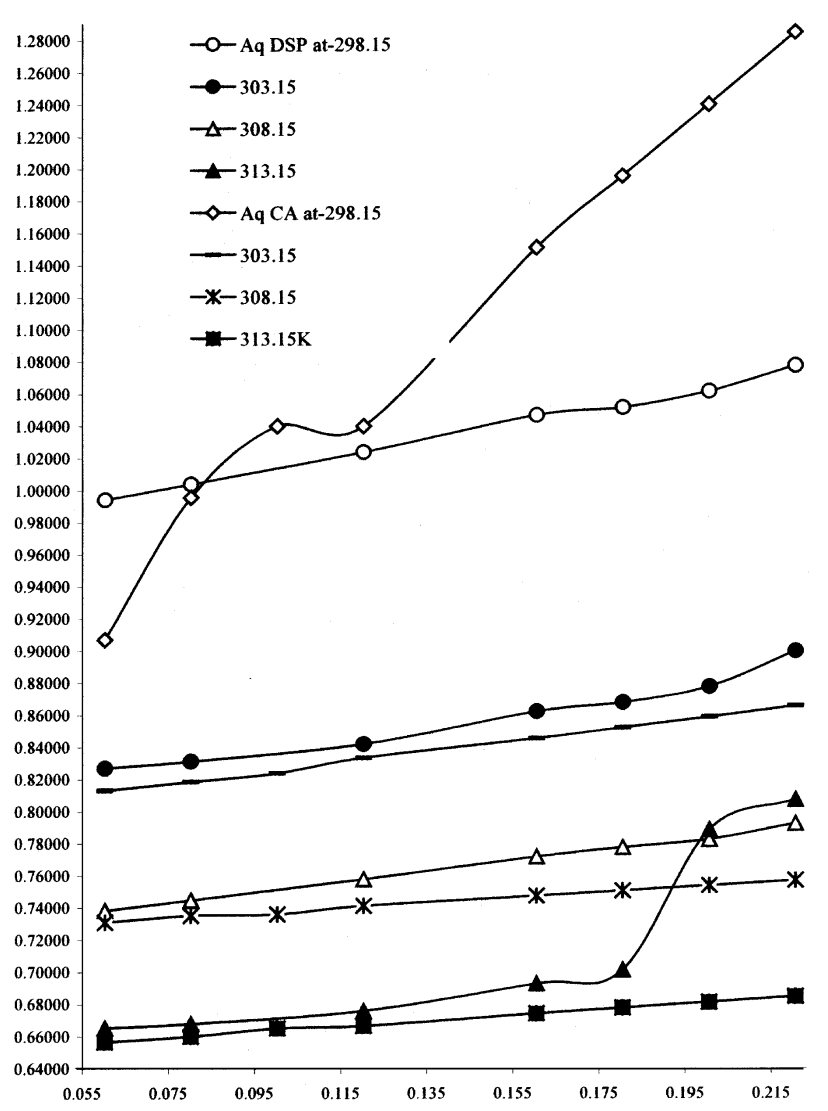

Figure 3. Viscosity vs $m$ of aqueous systems. 
(figure 1) and further increase in compositions decrease the $\rho$ values. However, compositions after 0.1355 of CA and $0.1544 \mathrm{~mol} \mathrm{~kg}^{-1}$ of DSP have higher $\rho$ values with slightly lower slopes. At 0.095 and $0.114 \mathrm{~mol} \mathrm{~kg}^{-1}$ both show bursting of micelles leading to decrease in $\rho$ but CA and DSP around $0 \cdot 1355$ and $0.1544 \mathrm{~mol} \mathrm{~kg}^{-1}$ respectively, again lead to micelle formation. This indicates that both mutually balance the behaviour of micelle bursting and thus, can buffer as the best pair for biological studies. For CA in varying concentrations of DSP, a maximum $\rho$ is seen for CA in $0.2006 \mathrm{~m}$ DSP and minimum for $0.1805 \mathrm{~m}$ DSP at 298.15 and $313.15 \mathrm{~K}$ respectively. The $\rho^{0}$ values of DSP and CA in aqueous solutions have been observed as a linear function of compositions of cosolute (figure 4). Thus densities are higher than that of the binary mixture by 0.00822 at each temperature and larger values for DSP in CA are reported than that for CA in DSP, implying that CA may not break down DSP-water interaction. $\mathrm{Na}^{+}$and $\mathrm{HPO}_{4}^{-2}$ ions may weaken electrostriction of water, which interacts with CA causing weaker $\mathrm{CA}$-water intermolecular forces. Likewise an increase in $\rho^{0}$ values of DSP from aq. to

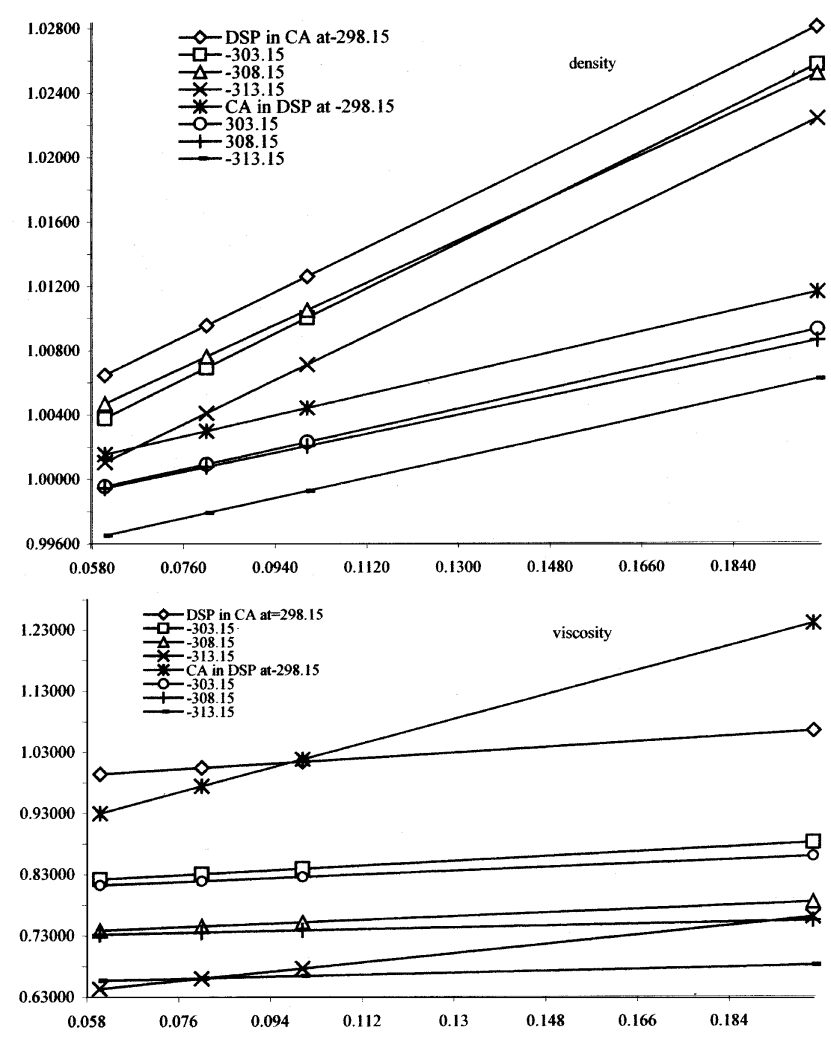

Figure 4. The $\rho^{\circ}$ vs $m$ of aqueous systems. varying temperatures of aq. CA solution is found as $0.00927,0.00942,0.00879,0.00916 / 10^{3} \mathrm{k} \mathrm{g} \mathrm{m}^{-3}$ at each temperature in $0.0605 \mathrm{~m} \mathrm{CA}$ and for $\mathrm{CA}$ in $0.0605 \mathrm{mDSP}$ as $0.00431,0.00417,0.00392$ and $0.00415 / 10^{3} \mathrm{~kg} \mathrm{~m}^{-3}$. Here, a larger increase is found for DSP in CA while it is lower for CA in DSP with considerable influence of CA on ion-water interactions but DSP has very little influence on molecular interactions of CA with water. The $\rho^{0}$ of DSP in CA with its composition is higher and proves stronger electrostatic forces between CA and water. But $\rho^{0}$ of DSP with varying compositions of CA increase by $0.00307,0.00312,0.0029$ and 0.00302 at 298.15, $303 \cdot 15,308 \cdot 15$ and $313 \cdot 15 \mathrm{~K}$ respectively and for CA with composition of DSP by $0.00142,0.0038$, $0 \cdot 00130$, and $0 \cdot 00138 / 10^{3} \mathrm{~kg} \mathrm{~m}^{-3}$. It implies that CA considerably increases $\rho^{0}$ of DSP while DSP slightly increases the $\rho$ values of CA. The $\eta^{0}$ values of DSP in aq. CA are higher than that of aq. DSP by $0.03001,0.02539,0.01975,0.04999$ and of CA in DSP than that of aq. CA by $0.13352,0.01986$, $0 \cdot 0094,0 \cdot 01042 / 0 \cdot 1 \mathrm{~kg} \mathrm{~m}^{-1} \mathrm{~s}^{-1}$. At $298 \cdot 15 \mathrm{~K}$ an increase in $\eta^{0}$ of DSP in 0.0605 to $0.0805 \mathrm{~m} \mathrm{CA}$ is noted as $0.00993,0.00982$ and 0.04873 and of CA in

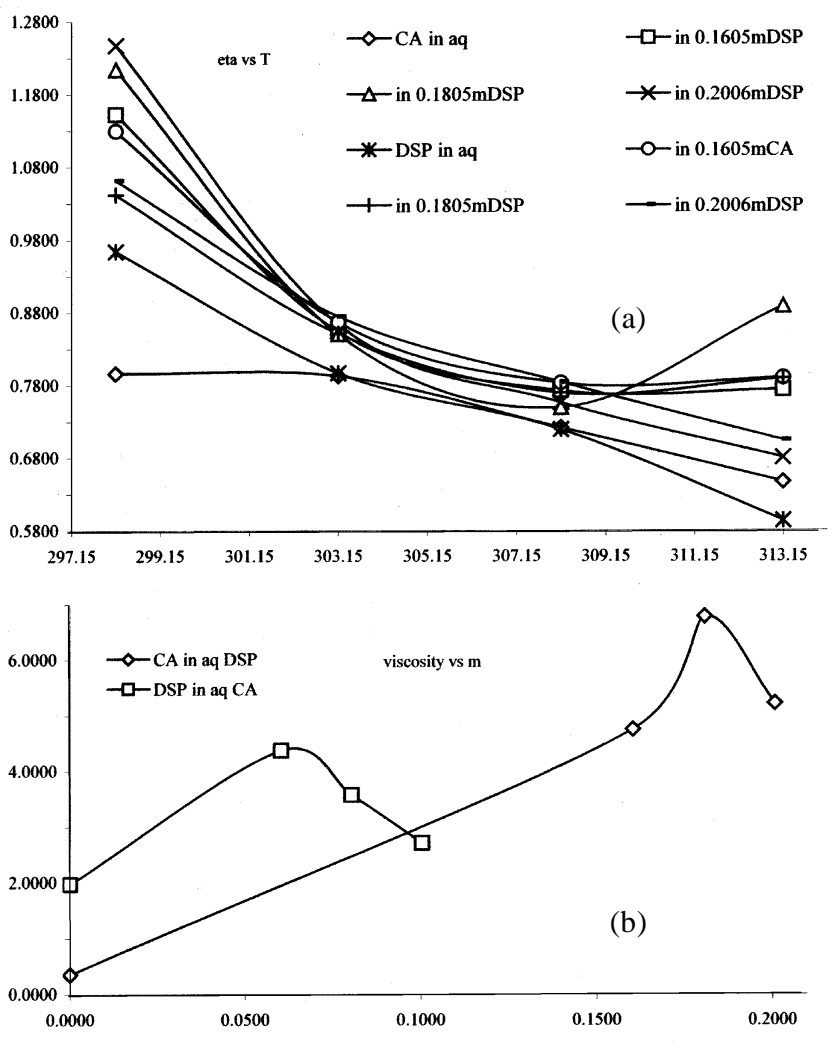

Figure 5. The $\eta^{0}$ vs TK of additive systems. (a) $\eta^{0}$ vs $m$ of additives and (b) $\rho^{0}$ vs $m$ systems. 
similar compositions of DSP as $0.04414,0 \cdot 04371$ and $0 \cdot 2214 / 0 \cdot 1 \mathrm{~kg} \mathrm{~m}^{-1} \mathrm{~s}^{-1}$ and at $303 \cdot 15,308 \cdot 15$ and $313.15 \mathrm{~K}$ still remain lower. At all DSP compositions, higher $\eta^{0}$ values of CA are found at all temperatures, with maximum effect of composition on $\mathrm{CA}$ viscosity. Perhaps the ions $\mathrm{Na}^{+}$and $\mathrm{HPO}_{4}^{2-}$ cause larger frictional forces in the viscous flow of CA solutions than for CA on DSP. An important feature observed in these systems is that micelle formation takes place for lower CA concentrations but higher concentrations of DSP. Interestingly, higher $V_{\phi}$ values are found at $303.15 \mathrm{~K}$ for both systems separately. Further higher $V_{\phi}^{0}$ values of CA in aq. DSP prove that ionic interaction of DSP seems to weaken electrostriction of water and intermolecular forces operating between CA and water molecules. The $\rho^{0}$ values of DSP in CA are higher by 0.00513/ $10 \mathrm{~kg} \mathrm{~m}^{-3}$ but, in general, $\rho^{0}$ of DSP in CA goes down for each composition with temperature. This indicates that molecular interaction of CA enhances electrostriction of water-DSP interaction. The $\rho^{0}$ values of DSP with composition in fixed compositions of CA and CA in DSP are a polynomial relation with compositions of DSP in CA and of CA in DSP. In general, $\eta$ values are found to be linear for aqueous binary systems as depicted in figure 3, however aq. CA and DSP at 298.15 and $313.15 \mathrm{~K}$ induce sharp increase in $\eta$ at lower and higher concentrations respectively. Figure 3 shows that $\eta$ value at each temperature is higher for aq. DSP than that for aq. CA indicating stronger interaction in the former. The $\eta$ values prove that electrostatic forces change in viscous flow and remain less effective. At 298.15 , the $\eta^{0}$ value is the lowest for aq. CA and is the highest for CA in $0 \cdot 2006,0 \cdot 1806$ and $0.1602 \mathrm{~m}$ of DSP. The $\eta^{0}$ values for the binary system converge to a common temperature point. The slope remains constant with respect to temperature. Values of $\eta^{0}$ and $\rho^{0}$ vs $m$ for ternary CA and DSP systems are given in figures $5 \mathrm{a}$ and $\mathrm{b}$. The $\eta^{0}$ values of DSP for $m \rightarrow 0 \mathrm{CA}$ are higher than that of CA for $m \rightarrow 0$ DSP. These values indicate stronger solute-cosolutesolvent interaction for DSP in CA solution compared to CA in DSP solution. However, the $\eta^{0}$ of DSP after $0.055 \mathrm{~m} \mathrm{CA}$ starts decreasing and continues up to $0 \cdot 1006 \mathrm{~m} \mathrm{CA}$ confirming that DSP may be involved in micelle formation at least around $0.055 \mathrm{~m}$ CA. Simultaneously the CA for 0 to $0.0157 \mathrm{~m} \mathrm{DSP}$ increases with almost the same slope values. The $\eta^{0}$ values of CA in DSP and of DSP in CA at $298.15 \mathrm{~K}$ are higher than those at other temperatures by
$0 \cdot 02267,0 \cdot 17234,0 \cdot 18578 / 0 \cdot 1 \mathrm{~kg} \mathrm{~m}^{-1} \mathrm{~s}^{-1}$ (figure 5a). These values show that at low temperature the compositions of DSP for CA and of CA for DSP systems cause stronger frictional forces, which resist the flow of an adjacent layer of solution. This effect continues for other compositions as well and may be attributed to cosolute-cosolute interactions. The $\eta^{0}$ values of DSP in $0.0602 \mathrm{~m} \mathrm{CA}$ are slightly higher than those of CA for $0.1605 \mathrm{~m}$ DSP at $298.15 \mathrm{~K}$ which confirms that molecular interactions of CA enhance the frictional force of DSP-water interactions more than that of DSP in CA-water. The $\eta^{0}$ values are regressed against temperature and coefficients are referred to $\eta_{\mathrm{T}}^{0}$, the limiting viscosity and $A_{T}$, slope constants at temperature approaching 0 value. The $\eta_{\mathrm{T}}^{0}$ of aq. DSP is higher than that of aq. CA by 1.6244 . The $\eta_{\mathrm{T}}^{0}$ of CA in aq. DSP are higher by $0.37759,3.2115$ and $2 \cdot 50658 / 0 \cdot 1 \mathrm{~kg} \mathrm{~m}^{-1} \mathrm{~s}^{-1}$ at $0.1605,1.805$ and $0.2005 \mathrm{~m}$ DSP. On transferring DSP to aq. CA, the $\eta^{0}$ drastically decreases to lower values than those of CA in aq. DSP. It implies that CA disrupts frictional forces of aq. DSP and this action dominates for $0.0605 \mathrm{~m} \mathrm{CA}$ but at higher concentrations of CA, it decreases. Similar trends are also observed for CA with compositions of DSP. The $A_{T}$ values are negative and of DSP in CA is higher than that of CA in aq. DSP, which proves that thermal energy decreases the frictional force. We note that higher compositions of CA result in lower $V_{\phi}^{0}$ values probably due to predominance of $\mathrm{CA}-\mathrm{CA}$ and CA-DSP interactions over CA-water and DSPwater interactions.

\section{Conclusion}

The $\rho^{0}$ values of aq. DSP decrease more with temperature compared to $\mathrm{CA}$, perhaps, due to weakening of electrostatic forces of hydrated $\mathrm{Na}^{+}$and $\mathrm{HPO}^{2+}$. Maximum value of $\rho$ of DSP in $0.0910 \mathrm{~mol} \mathrm{~kg}^{-1} \mathrm{CA}$ solution in water and $0.114 \mathrm{~mol} \mathrm{~kg}^{-1}$ DSP with positive and negative slopes before and after these compositions denote bursting and reorganization of micelles respectively. Higher densities for ternary systems than those for binary, with larger values for DSP in aqueous solutions of CA than that of CA in aqueous solution of DSP show CA weakens electrostriction of water. Higher $\rho^{0}$ for DSP in CA solution shows stronger electrostatic forces between $\mathrm{CA}$ and water. The higher $\eta^{0}$ of DSP in aq. CA than that in water alone of CA in aq. DSP than that of CA in water implies larger frictional forces in viscous flows of 
CA and DSP solutions due to the presence of $\mathrm{Na}^{+}$and $\mathrm{HPO}_{4}^{2-}$. The lower $V_{\phi}$ of aq. DSP compared to that of aq. CA proves greater internal pressure for ionic interactions in DSP solution. The $V_{\phi}^{0}$ values converge to a particular composition due to micelle formation and higher $V_{\phi}^{0}$ values of CA in aq. DSP prove weakening of water and CA-water electrostrictions. The higher $\eta$ values of aq. DSP compared to that of aq. CA is attributed to electrostatic forces on flow, converging $\eta^{0}$ values of the binary system to a fixed temperature. The higher $\eta^{0}$ values of DSP for $m \rightarrow 0$ CA compared to that of CA for $m \rightarrow 0$ DSP, depict stronger solute-cosolute-solvent interaction for DSP in CA than that of CA in DSP.

\section{Acknowledgements}

The author thanks the Department of Science and Technology, Govt. of India for financial support and Mr S P Meena for fruitful discussions.

\section{References}

1. Barone G, Castronuovo G, Del Vecchio P, Elia V and Puzziello S 1989 J. Solution Chem. 181105

2. Barone G, Castronuovo G, Elia V, Giancola C and Puzziello S 1990 J. Solution Chem. 19855

3. Key J and Weitzmann P P J (eds) 1987 Kreb's citric acid cycle: Half a century and still turning. Biochemi- cal Society Symposium 54 (London: The Biochem. Soc.)

4. Hellewege K H (eds) 1977 Densities of liquid systems and their heat capacities. Landolt-Bornstein numerical data and functional relationship in science and technology (New Series) (Berlin: SpringerVerlag) pp 47-48 and 80-81

5. Franks F (ed.) 1972-1983 Water a comprehensive treatise (New York: Plenum) vol 1-7

6. Chicard K and Skinner J F 1969 J. Phys. Chem. 73 2060

7. Ben-Naim A 1989 J. Chem. Phys. 907412

8. Ben-Naim A 1990 Biopolymers 29567

9. Masson D O 1929 Philos. Mag. 8218

10. Jones G and Dole M 1929 J. Am. Chem. Soc. 512950

11. Falkenhagen H and Dole M 1929 Phys. Z. 30611

12. Barry R B and Irving F M 1970 J. Phys. Chem. 741056

13. Tamak K, Ohara Y and Isomura Y 1973 Bull. Chem. Soc. Jpn 46289

14. Pandey J D, Akhtar Y and Sharma A K 1998 Indian J. Chem. A37 1094

15. Parmar M L and Dhimniand D K 2002 J. Chem. Soc. 9729

16. Ali A, Kumar N and Ibrahim M 2002 Proc. Indian Sci. (Chem. Sci.) 114495

17. Man Singh 2001 J. Indian Chem. Soc. 78397

18. Apelblat A and Manzurola E 1999 J. Chem. Thermodyn. 31869

19. Levitt B P and Kitchener J A 1954 Findlay's practical physical chemistry 9th edn (London and New York: Longman) pp. (a) 6-23, (b) p. 430, (c) p. 90

20. Man Singh 2003 J. Indian Chem. Soc. 80704

21. Mochizuki S and Wakisaks A 2002 J. Phys. Chem. A106 5095 